Original Research

\title{
Twelve-Week Quadriceps Strength as A Predictor of Quadriceps Strength At Time Of Return To Sport Testing Following Bone- Patellar Tendon-Bone Autograft Anterior Cruciate Ligament Reconstruction
}

Joseph P Hannon, PT, DPT, PhD, SCS ${ }^{1}$, Sharon Wang-Price, PT, PhD, COMT, FAAOMPT ${ }^{2}$, Shiho Goto, PhD, ATC ${ }^{1}$ Steven Singleton, $\mathrm{MD}^{3}$, Lindsey Dietrich, MD ${ }^{4}$, James Bothwell, $\mathrm{MD}^{5}$, Curtis Bush, $\mathrm{MD}^{6}$, Craig Garrison, PT, PhD, ATC

${ }^{1}$ Texas Health Sports Medicine, ${ }^{2}$ School of Physical Therapy, Texas Woman's University, ${ }^{3}$ Texas Health Orthopedic Specialists, ${ }^{4}$ Sideline Orthopedics and Sports, ${ }^{5}$ Fort Worth Orthopedics, ${ }^{6}$ Texas Orthopedic Associates, ${ }^{7}$ Department of Physical Therapy \& Human Movement Sciences: Feinberg School of Medicine, Northwestern University

Keywords: movement system, return to sport, quadriceps strength, anterior cruciate ligament reconstruction

https://doi.org/10.26603/001c.23421

\section{International Journal of Sports Physical Therapy}

Vol. 16, Issue 3, 2021

\section{Background}

Restoration of quadriceps strength following anterior cruciate ligament reconstruction (ACL-R) continues to challenge both patients and clinicians. Failure to adequately restore quadriceps strength has been linked to decreased patients' self-reported outcomes and an increased risk for re-injury. Early identification of quadriceps strength deficits may assist in tailoring early interventions to better address impairments.

\section{Purpose}

The purpose of this study was to assess the relationship between early (12 weeks following ACL-R) isokinetic peak torque and isokinetic peak torque at time of return to sport (RTS) testing.

\section{Study Design}

Cohort Study

\section{Methods}

A total of 120 participants (males $=55$; females $=65$ ) were enrolled in the study (age $=$ $16.1 \pm 1.4$ yrs; height $=1.72 \pm 10.5 \mathrm{~m}$; mass $=70.7 \pm 16.3 \mathrm{~kg}$ ). All participants were level 1 or 2 cutting and pivoting sport athletes who underwent a primary bone-patellar tendon-bone autograft ACL-R. Participants were tested at two time points: 12 weeks following surgery and again at time of RTS testing. A linear regression model was carried out to investigate the relationship between age, sex, and isokinetic peak torque at 12 weeks following ACL-R and isokinetic peak torque at time of RTS testing.

\section{Results}

When 12-week isokinetic peak torque was entered first for the hierarchy regression analysis, this factor was predictive of the peak torque at the time of RTS testing, $F(1,118)$ $=105.6, p<0.001, R^{2}=0.472$, indicating that the 12-week quadriceps strength accounted for $47 \%$ of the variance in the quadriceps strength at the time of RTS testing. When age and sex were added in the regression analysis, both factors only added $0.8 \%$ of variance for the quadriceps strength at the time of RTS testing.

\footnotetext{
Corresponding Author:

Joseph Hannon PT, DPT, PhD, SCS CSCS

6301 Harris Pkwy, Suite 150

Fort Worth, TX 76102

phone: 817-433-8206

email: JosephHannon@texashealth.org
} 


\section{Conclusion}

Isokinetic peak torque at 12 weeks following surgery was shown to be a significantly strong predictor (47\%) for isokinetic quadriceps strength recovery at time of RTS. This finding underscores the importance of early restoration of quadriceps strength and that while non-modifiable factors such as sex and age are important, early restoration of quadriceps strength most strongly influences late stage quadriceps strength.

\section{Level of Evidence}

\section{INTRODUCTION}

Restoration of quadriceps strength following anterior cruciate ligament reconstruction (ACL-R) continues to challenge both patients and clinicians. ${ }^{1-4}$ Quadriceps weakness following ACL-R, particularly with bone-patellar tendon-bone (BPTB) autograft, has been linked to poor self-reported outcomes, ${ }^{4-6}$ altered biomechanics, ${ }^{1,7}$ and increased risk of second injury. ${ }^{8}$ These deficits in quadriceps strength following ACL-R seem to be more pronounced in those individuals undergoing BPTB autografts versus other graft types (i.e. hamstring autograft). ${ }^{4}$ Conversely, restoration of quadriceps strength has been shown to be protective against second ACL injury. ${ }^{3,9-11}$ Additionally, this prolonged and persistent quadriceps weakness has been theorized to contribute to the early posttraumatic osteoarthritis seen after ACL-R. ${ }^{12,13}$ Thus, restoring quadriceps strength is crucial for the short- term and long-term health of individuals following ACL-R.

The development of early objective criteria that can identify patients who have restored sufficient quadriceps strength can assist clinicians in appropriately tailoring early interventions. In the changing healthcare environment, many patients have limited access to physical therapy and the ability to identify patients who are on, ahead, or behind schedule early in the rehabilitation process may allow the clinician to direct care based upon objective data with the goal of improved patient outcomes. Several such early measures have been established for other impairments for patients after ACL-R. For example, Garrison et al. ${ }^{14}$ found a significantly positive relationship between the Y-Balance Test-Lower Quarter (YBT-LQ) at 12-weeks post-operatively and jump performance at time of return to sport (RTS). Participants who demonstrated a greater than $4 \mathrm{~cm}$ YBTLQ anterior reach difference between sides at 12-weeks did not meet criteria (more than $10 \%$ difference) at time of RTS testing for the single and triple hop testing. ${ }^{14}$ Lablanca et al. ${ }^{15}$ found that ground reaction force symmetry during a squat at one-month post-operation was an independent predictor $\left(\Delta R^{2}=0.14, p<0.01\right)$ of ground reaction force symmetry during a landing task at the time of RTS. Sigward et al. found that knee moments during gait at four weeks were significantly related to knee moments during running at four months. ${ }^{16}$ These studies highlight the importance of how an early objective measurement of an impairment can be related to the outcome of a similar impairment domain in a later stage of recovery and ultimately influence criteria used to determine when an athlete is ready to return to play. $3,4,17,18$

While numerous studies have found correlations be- tween late-stage isokinetic quadriceps strength and other objective measures (patient reported outcomes, hop testing, balance testing), little focus has been given to early quadriceps strength, such as that at 12 weeks. $1,3,9,11,19$ However, previous data suggests that isometric quadriceps strength increases longitudinally at 4,8 , and 12 weeks following ACL-R. ${ }^{20,21}$ This early time frame is important in rehabilitation as this is when patients are beginning to transition to running- and plyometric based training training. ${ }^{22,23}$ Additionally, It has been well documented that a significant deficit in quadriceps strength is still present at this time ${ }^{23}$ however, no studies have quantified this deficit or correlated this early deficit with late stage impairment. At 12-weeks after ACL-R many patients are still under the direct care of their rehab specialist and early identification of quadriceps strength deficits may allow modification to the treatment plan to address said deficit. Furthermore, utilizing these early quadriceps strength measures to help predict late stage strength impairments can assist in clinical decision making.

Therefore, the purpose of this study was to examine the relationship between isokinetic peak torque of individuals at 12-weeks following ACL-R and isokinetic peak torque of the same individuals at time of RTS testing. In addition, the secondary purpose of the study was to determine if isokinetic knee extension peak torque at 12 weeks would predict quadriceps strength recovery at the time of RTS testing. It was hypothesized that isokinetic peak torque at 12 weeks would be highly predictive to isokinetic peak torque at time of RTS testing.

\section{METHODS}

A total of 120 participants who met the inclusion criteria were enrolled in the study. Table 1 displays the characteristics of the participants. All participants were screened by the study staff prior to enrollment. Participants were considered eligible if they injured their ACL for the first time, were level 1 or 2 athletes who planned on returning to competitive sport and did not have any full thickness chondral injuries or grade II or III medial collateral ligament (MCL), lateral collateral ligament (LCL) or posterior collateral ligament (PCL) injuries. All participants gave informed consent to participate and the rights of each person were protected. If the participant was a minor, parental consent and child assent were attained. The Institutional Review Board of Texas Health Resources approved this research study.

All participants completed two testing sessions with the first session occurring at 12 weeks following ACL-R. The second testing session was at the time of RTS testing which 
Table 1: Characteristics of the participants $(n=120)$

\begin{tabular}{lc} 
Height $(\mathrm{cm})$ & $172.0 \pm 10.5$ \\
Weight $(\mathrm{Kg})$ & $70.7 \pm 16.3$ \\
Age (years) & $16.1 \pm 1.4$ \\
Gender & $\mathrm{M}=55, \mathrm{~F}=65$ \\
Mechanism of injury (contact/indirect/non-contact) & $23 / 24 / 73$ \\
Time from surgery to RTS testing (months) & $7.3 \pm 1.3$ \\
\hline
\end{tabular}

RTS = return to sport

Table 2: Descriptive statistics (means \pm SD) of clinical tests and knee extensor peak torques of all participants at 12 weeks following ACL-R and at the time of return-to-sport (RTS) $(n=120)$

\begin{tabular}{lccc}
\hline & 12 weeks & RTS & $p$-value \\
\hline IKDC Score (\%) & $66.86 \pm 10.77$ & $90.52 \pm 11.25$ & $<0.001$ \\
Knee Extension ROM ( $\left.{ }^{\circ}\right)$ & & & \\
$\quad$ Involved & $1.73 \pm 2.34$ & $2.38 \pm 2.33$ & 0.045 \\
$\quad$ Uninvolved & $4.37 \pm 1.22$ & $3.29 \pm 2.22$ & 0.320 \\
Knee Flexion ROM ( $\left.{ }^{\circ}\right)$ & & & 0.258 \\
$\quad$ Involved & $139.60 \pm 9.38$ & $140.73 \pm 12.03$ & 0.703 \\
$\quad$ Uninvolved & $143.27 \pm 14.86$ & $143.22 \pm 7.30$ & $\mathrm{~N} / \mathrm{A}$ \\
Single hop distance LSI (\%) & $\mathrm{N} / \mathrm{A}$ & $95.80 \pm 5.73$ & $\mathrm{~N} / \mathrm{A}$ \\
Triple Hop Distance LSI (\%) & $\mathrm{N} / \mathrm{A}$ & $95.95 \pm 11.99$ & \\
Y-Balance Test composite score (\%) & & $95.71 \pm 9.00$ & $<.001$ \\
$\quad$ Involved & $91.51 \pm 6.92$ & $99.62 \pm 5.39$ & 0.334 \\
$\quad$ Uninvolved & $96.54 \pm 6.76$ & $1.55 \pm 0.45$ & $<0.001$ \\
Knee Extensor Peak Torque (Nm/Kg) & $1.13 \pm 0.40$ & & \\
\hline
\end{tabular}

IKDC = International Knee Documentation Committee Questionnaire, ROM= range of motion; LSI= limb symmetry index.

was an average of $7.2 \pm 1.3$ months following surgery. Decision to complete RTS testing was based on input from the surgeon and physical therapist based on the participant's clinical presentation. Both testing sessions consisted of isokinetic isokinetic peak torque testing on the Biodex MultiJoint Testing and Rehabilitation System (Biodex Medical Systems, Shirley, NY). Isokinetic peak torque was assessed bilaterally. The femoral condyle of the tested limb was aligned with the Biodex axis of rotation following the manufacturer's instructions. Participants performed five repetitions of submaximal knee extension/flexion to familiarize themselves with the testing motion. To determine isokinetic peak torque, participants performed five consecutive concentric contractions at $60^{\circ} / \mathrm{sec}$ on each limb. ${ }^{24}$ The uninvolved limb of participants was tested prior to testing the involved limb. The average of the five trials was normalized to each participant's body weight and used for analysis. Any testing that resulted in a coefficient of variance of greater than $15 \%$ was discarded and the quadriceps strength test was repeated following an appropriate rest interval. Additional clinical test results, including the International Knee Documentation Committee Questionnaire (IKDC) scores, range of motion (ROM) of knee extension and flexion, limb symmetry indices (LSI) of single hop distance, triple hop distance, YBT-LQ composite scores, collected at 12 weeks following ACL-R and at the time of RTS testing, were used to describe the participants of the study, and were not used for statistical analysis (Table 2).

\section{STATISTICAL ANALYSIS}

Descriptive statistics were calculated for participant characteristics and normalized quadriceps strength of all participants. All statistical analyses were performed using SPSS version 23 (IBM, Armonk, NY). First, a Pearson-ProductMoment correlation coefficient was calculated between isokinetic peak torque at 12 weeks following ACL-R and isokinetic peak torque at time of RTS testing. Next, a regression was conducted to determine the influence of quadriceps strength at time of RTS testing in addition to age and sex which have been shown to be significant predictors of quadriceps strength. ${ }^{25,26}$ The alpha level was set at 0.05 for all statistical analyses. 
Table 3: Hierarchical regression analysis results of 12-week extensor peak torque, age and sex on extensor peak torque at time of return-to-sport $(\mathrm{RTS})$ testing $(\mathrm{n}=120)$

\begin{tabular}{|c|c|c|c|c|c|c|}
\hline & $\mathbf{B}$ & $\mathbf{R}^{2}$ & $\boldsymbol{\Delta} \boldsymbol{R}^{2}$ & $\boldsymbol{B}$ & $\boldsymbol{\beta}$ & $\boldsymbol{\beta}$ \\
\hline Step 1 & & & & & & \\
\hline 12-week Extensor Peak Torque & & 0.472 & & 0.781 & 0.687 & $<0.001^{*}$ \\
\hline Step 2 & & 0.480 & 0.008 & & $<0.001^{*}$ \\
\hline 12-week Extensor Peak Torque & & & & 0.744 & 0.655 & $<0.001^{*}$ \\
\hline Age & & & & -0.013 & -0.042 & 0.545 \\
\hline Sex (0=male, 1=female) & & & & -0.084 & -0.093 & 0.210 \\
\hline
\end{tabular}

B: constant; $\Delta R^{2}: R^{2}$ change; $B=$ unstandardized coefficients; $\beta=$ standardized coefficients.

\section{RESULTS}

The correlation showed a significant strong positive relationship (Figure 1) between quadriceps strength at 12 weeks after ACL-R and quadriceps strength at the time of RTS ( $r=$ $0.687 ; p<0.001$ ). Prior to the regression analysis, data were checked for violations of the assumptions for regression analysis. A normal predicted probability (P-P) plot showed that the standardized residuals were normally distributed, thus meeting the normality assumption. The scatterplot of standardized predicted values versus standardized residuals showed that the data met the assumptions of homoscedasticity and linearity.

Next, a hierarchical regression was conducted for quadriceps strength at the time of RTS with 12-week quadriceps strength entered in the first step, and age and sex the second step (Table 3). The regression analysis showed that 12-week quadriceps peak torque was predictive of quadriceps peak torque at the time of RTS testing, $F(1,118)=$ 105.6, $p<0.001, R^{2}=0.472$, indicating that 12 -week quadriceps peak torque could explain $47 \%$ of the variance in quadriceps peak torque at the time of RTS testing. However, after age $(\beta=-0.042, p=0.545)$ and $\operatorname{sex}(\beta=-0.093, p=$ 0.210 ) were added in the second step, these two factors only added $0.8 \%$ of variance for the quadriceps peak torque at the time of RTS testing, $F(3,116)=35.7, p<0.001, R^{2}=0.480$, $\Delta R^{2}=0.008$. In contrast to what previous literature has shown, ${ }^{25}$ this result suggests that age and sex do not appear to be significant predictors of the quadriceps strength development at the time of RTS testing. Therefore, only 12-week quadriceps peak torque was retained in the prediction equation: Predicted quadriceps peak torque at the time of RTS testing $(\mathrm{Nm} / \mathrm{kg})=0.666+0.781 \times 12$-week peak torque $(\mathrm{Nm} / \mathrm{kg})$.

\section{DISCUSSION}

The purpose of this study was to examine the relationship between isokinetic peak torque at an early stage (i.e., 12 weeks) of recovery from ACL-R and at the time of RTS testing (i.e., 7-9 months after ACL-R). The 12-week quadriceps strength was shown to be a strong predictor, and that $47 \%$ of isokinetic peak torque at the time of RTS testing was explained by the isokinetic peak torque at 12 -weeks after ACL-

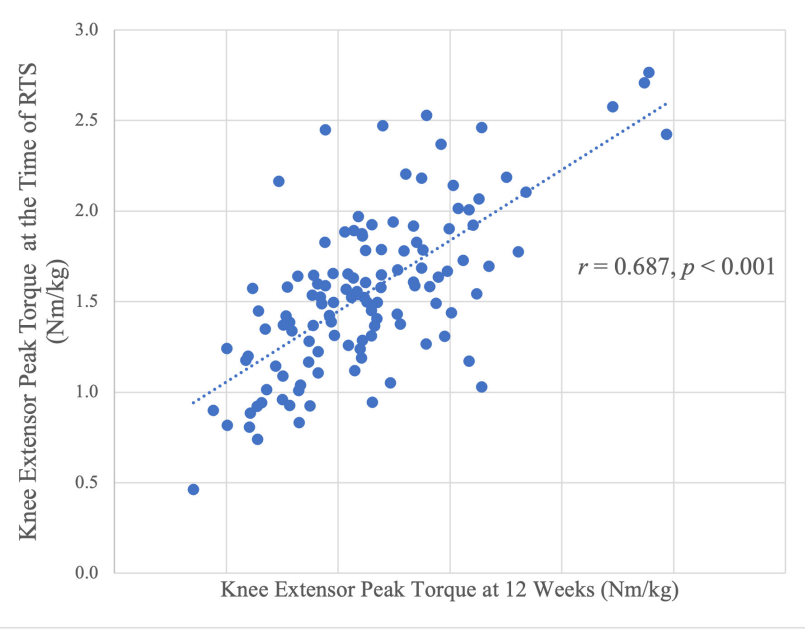

Figure 1: Scatter plot of knee extensor peak torque $(\mathrm{Nm} / \mathrm{Kg})$ of the quadriceps muscle at 12 weeks and at the time of return-to-sport $(\mathrm{RTS})(\mathrm{n}=120)$

R. The identification of isokinetic peak torque deficits at this early stage of ACL-R recovery can further provide clinicians with an understanding of quadriceps strength recovery progression during the rehabilitation process. As restoring quadriceps strength continues to challenge surgeons, clinicians, and patients, the short- and long-term consequences of not restoring quadriceps strength can be significant, including re-injury and potentially life-style altering post-traumatic osteoarthritis. ${ }^{9,13,27}$ Numerous studies have demonstrated that at the time of RTS testing, many individuals continue to demonstrate deficits in quadriceps strength.3,4,8,19 While this is certainly problematic, for many clinicians and patients the identification of these deficits at this point may be too late for effective intervention. In most cases by six months post-operation, patients have typically transitioned out of structured care; therefore, early identification of these strength deficits may assist clinicians in better tailoring early interventions for quadriceps strength deficits. When available, the use isokinetic testing at 12 weeks and the regression equation provided in this manuscript can help to provide clinicians an indication of how a patients quadricep is progressing. 
Contrary to other reports in the literature, ${ }^{25,26}$ age and gender were found to be weak predictors for quadriceps strength recovery at the time of RTS. As the majority of our study participants were high school and freshmen/sophomore college athletes, the narrow age range could have contributed to the non-significant finding. Recently, Kuenze et al. ${ }^{25}$ found that female athletes had slower quadriceps strength recovery at the time of RTS after ACL-R than male athletes. However, in this present study no difference was found between female and male athletes. The difference between the studies could in part be explained by the inclusion of all graft types (BPTB, hamstrings and allograft) in the study by Kuenze et al. In addition, only $10 \%$ of Kuenze et al.'s male participants had a BPTB graft with their ACL-R, whereas $39 \%$ of their female participants had a BPTB graft. This disproportionate percentage of participants with BPTB grafts, also may have influenced the sex-difference results of their study.

Few studies have examined isokinetic peak torque in the early stage of recovery (i.e., 12 weeks or earlier) following ACL-R. Harput et al. reported on 24 subjects following ACL$\mathrm{R}$ who completed isometric quadriceps strength testing at 4,8 , and 12 weeks following surgery. ${ }^{21}$ As expected, they found a longitudinal increase in quadriceps strength across time points. Similarly, as part of studies examining early neuromuscular rehabilitation, Dreschler et al. and Fitzgerald et al. reported on isometrics quadriceps strength at 90 and 60 degrees of knee flexion respectively. Dreschler et al. $^{20}$ tested subjects at 4 - and 12-weeks following ACL-R, and Fitzgerald et al. ${ }^{28}$ completed testing at 12 -weeks and 16 -weeks post ACL-R. Both studies also showed an increase in strength across time. These studies help to support the notion of early isokinetic peak torque testing and that strength generally increases across time in these early phases following ACL-R. Furthermore, Hartigan et al. previously demonstrated that pre-operative quadriceps strength, along with patient age, correctly predicted $82 \%$ of subjects who did not pass RTS criteria at time of RTS testing. Interestingly, Hallagin et al. compared pre-operative quadriceps strength to strength at 12 -weeks post ACL-R, and found a $44 \%$ decrease in quadriceps strength between these two time points. ${ }^{23}$ These findings together seem to suggest that the additional trauma of surgery has a significant deleterious effect on early quadriceps strength and that while preoperative strength strongly influences strength at time of RTS, the progression of strength is not linear in nature. ${ }^{29}$ Comparison across these studies is difficult, as some studies were intervention studies and utilized isometric testing at 60,70 , and 90 degrees of knee flexion, while the study by Hallagin et al. ${ }^{23}$ utilized isokinetic testing.

A limitation of these studies examining the quadriceps strength in the early stage of rehabilitation is that there is limited follow-up after ACL-R. It is well accepted that with the appropriate treatment approaches, strength will increase in the early phases after surgery. However, given the significant quadriceps strength deficits that are reported at 6,12 and 24 months after ACL-R the progression of strength is slow and clearly not linear. Thus, how these early strength measures predict long term (greater than six months post-surgery) outcomes is relatively unknown. Pua et $\mathrm{al}^{18}$ reported on a cohort of 70 patients who completed isometric quadriceps strength testing at 70 degrees of knee flexion at 6 weeks following ACL-R. In their multivariate regression model, quadriceps strength at six weeks was a significant predictor of six-month single-leg hop for distance and single leg vertical countermovement jump. ${ }^{18}$ Similarly, Beischer et al. found that $40 \%$ of the athletes in their study who had a limb symmetry indices of $80 \%$ or higher at four months following ACL-R achieved symmetrical muscle function at 12 months compared with fewer than $10 \%$ of the athletes who had an limb symmetry index (LSI) lower than $80 \%$. ${ }^{30}$ The findings from Pua et al. and Beischer et al. support the findings from our study that quadriceps strength in the early stage of post-operative ACL- $R$ has significant implications on late stage objective measures.

Early objective strength values have the potential to be invaluable to patients and clinicians. In the changing healthcare landscape with many patients facing high copayments and limited approved physical therapy visits, access to long-term physical therapy following ACL-R is becoming increasingly limited. The ability to identify patients who are on, ahead, or behind schedule early in the process will allow more directed early treatment and hopefully improved patient outcomes. The results of this study indicated that $48 \%$ of isokinetic quadriceps strength at time of RTS testing was explained by 12 -weeks quadriceps strength, age and sex. There are likely many factors that help explain the remaining $52 \%$ of strength at RTS including pain, inflammation, swelling, fear, atrophy, and decreased neural drive. ${ }^{31}$ Identification of the additional factors that contribute to quadriceps strength at time of RTS is the obvious next step to allow for more directed treatment strategies.

This study is not without limitations. The primary limitation of this study is that individuals were not assessed for meeting RTS criteria at time of RTS testing. This study is part of a larger ongoing ACL outcomes study and the purpose of this sub investigation was to assess for the specific relationship between 12-week and RTS isokinetic peak torque values, not to assess if 12 -week strength values could differentiate those who returned or did not return to sport. Additionally, there was no follow up beyond RTS in this cohort and so there is currently not a definitive way to determine if/when these athletes returned to sport following testing. There is also a limitation in the generalizability of this study as only those athletes following primary ACL-R with PBTB graft type were included and these findings likely change when other graft types are included. Thirdly, no measurement of central activation ratio (a ratio that measures maximum voluntary force produced divided by the force produced following a superimposed burst during a maximal voluntary contraction) was taken and this has been shown to be diminished following ACL-R and play a major role in quadriceps strength. ${ }^{5}$ Lastly, there are some inherent limitations with using isokinetic dynamometry when considering clinical applicability; however, it remains the gold standard for measurement of strength following ACL-R.

\section{CONCLUSIONS}

In conclusion, quadriceps strength at 12 weeks following surgery was shown to be a significantly strong predictor 
(47\%) for isokinetic quadriceps strength recovery at time of RTS. This finding underscores the importance of early restoration of quadriceps strength and that while non-modifiable factors such as sex and age are important, early restoration of quadriceps strength most strongly influences late stage quadriceps strength.

\section{CONFLICT OF INTERESTS}

The authors have no conflicts of interest to declare.

Submitted: May 26, 2020 CDT, Accepted: January 04, 2021 CDT 


\section{REFERENCES}

1. Blackburn JT, Pietrosimone B, Harkey MS, Luc BA, Pamukoff DN. Quadriceps function and gait kinetics after anterior cruciate ligament reconstruction. Med Sci Sports Exerc. 2016;48(9):1664-1670.

2. Hannon J, Wang-Price S, Goto S, Garrison JC, Bothwell JM. Do muscle strength deficits of the uninvolved hip and knee exist in young athletes before anterior cruciate ligament reconstruction? Orthop J Sports Med. 2017;5(1):2325967116683941.

3. Zwolski C, Schmitt LC, Quatman-Yates C, Thomas $S$, Hewett TE, Paterno MV. The influence of quadriceps strength asymmetry on patient-reported function at time of return to sport after anterior cruciate ligament reconstruction. Am J Sport Med. 2015;43(9):2242-2249.

4. Lepley LK. Deficits in quadriceps strength and patient-oriented outcomes at return to activity after ACL reconstruction: a review of the current literature. Sports Health. 2015;7(3):231-238.

5. Hart JM, Pietrosimone B, Hertel J, Ingersoll CD. Quadriceps activation following knee injuries: a systematic review. J Ath Train. 2010;45(1):87-97.

6. Pietrosimone B, Lepley AS, Harkey MS, et al. Quadriceps strength predicts self-reported function post-ACL reconstruction. Med Sci Sports Exerc. 2016;48(9):1671-1677.

7. Garrison JC, Hannon J, Goto S, Giesler L, Bush C, Bothwell JM. Participants at three months postoperative anterior cruciate ligament reconstruction (ACL-R) demonstrate differences in lower extremity energy absorption contribution and quadriceps strength compared to healthy controls. Knee. 2018;25(5):782-789.

8. Paterno MV, Rauh MJ, Thomas S, Hewett TE, Schmitt LC. Quadricep femoris strength at return to sport identifies limb at increased risk of future ACL injury after ACL reconstruction in young athletes. Orthop J Sports Med. 2019;7:2325967119S2325900329.

9. Grindem H, Snyder-Mackler L, Moksnes H, Engebretsen L, Risberg MA. Simple decision rules can reduce reinjury risk by $84 \%$ after ACL reconstruction: the Delaware-Oslo ACL cohort study. Br J Sports Med. 2016;50(13):804-808.

10. Padua DA, DiStefano LJ. Sagittal plane knee biomechanics and vertical ground reaction forces are modified following ACL injury prevention programs: a systematic review. Sports Health. 2009;1(2):165-173.
11. Ithurburn MP, Altenburger AR, Thomas S, Hewett TE, Paterno MV, Schmitt LC. Young athletes after ACL reconstruction with quadriceps strength asymmetry at the time of return-to-sport demonstrate decreased knee function 1 year later. Knee Surg Sports Traumatol Arthrosc. 2018;26(2):426-433.

12. Øiestad B, Juhl C, Eitzen I, Thorlund J. Knee extensor muscle weakness is a risk factor for development of knee osteoarthritis. A systematic review and meta-analysis. Osteoarthr Cartil. 2015;23(2):171-177.

13. Pietrosimone B. Understanding, detecting, and managing the risk of posttraumatic osteoarthritis following anterior cruciate ligament reconstruction in the military. N C Med J. 2017;78(5):327-328.

14. Garrison JC, Bothwell JM, Wolf G, Aryal S, Thigpen CA. Y balance test ${ }^{\mathrm{TM}}$ anterior reach symmetry at three months is related to single leg functional performance at time of return to sports following anterior cruciate ligament reconstruction. Int J Sports Phys Ther. 2015;10(5):602.

15. Labanca L, Laudani L, Menotti F, et al. Asymmetrical lower extremity loading early after anterior cruciate ligament reconstruction is a significant predictor of asymmetrical loading at the time of return to sport. Am J Phys Med Rehabil. 2016;95(4):248-255.

16. Sigward SM, Lin P, Pratt K. Knee loading asymmetries during gait and running in early rehabilitation following anterior cruciate ligament reconstruction: a longitudinal study. Clin Biomech. 2016;32:249-254.

17. Eitzen I, Holm I, Risberg MA. Preoperative quadriceps strength is a significant predictor of knee function two years after anterior cruciate ligament reconstruction. Br J Sports Med. 2009;43(5):371-376.

18. Pua Y-H, Mentiplay BF, Clark RA, Ho J-Y. Associations among quadriceps strength and rate of torque development 6 weeks post anterior cruciate ligament reconstruction and future hop and vertical jump performance: a prospective cohort study. $J$ Orthop Sports Phys Ther. 2017;47(11):845-852.

19. Barfod KW, Feller JA, Hartwig T, Devitt BM, Webster KE. Knee extensor strength and hop test performance following anterior cruciate ligament reconstruction. The Knee. 2019;26(1):149-154. 
20. Drechsler WI, Cramp MC, Scott OM. Changes in muscle strength and EMG median frequency after anterior cruciate ligament reconstruction. Eur J Appl Physiol. 2006;98(6):613-623.

21. Harput G, Kilinc HE, Ozer H, Baltaci G, Mattacola CG. Quadriceps and hamstring strength recovery during early neuromuscular rehabilitation after ACL hamstring-tendon autograft reconstruction. J Sport Rehabil. 2015;24(4):398-404.

22. Adams D, Logerstedt D, Hunter-Giordano A, Axe MJ, Snyder-Mackler L. Current concepts for anterior cruciate ligament reconstruction: a criterion-based rehabilitation progression. J Orthop Sports Phys Ther. 2012;42(7):601-614.

23. Hallagin C, Garrison JC, Creed K, Bothwell JM, Goto S, Hannon J. The relationship between preoperative and twelve-week post-operative y-balance and quadriceps strength in athletes with an anterior cruciate ligament tear. Int J Sports Phys Ther. 2017;12(6):986

24. Cvjetkovic DD, Bijeljac S, Palija S, et al. Isokinetic testing in evaluation rehabilitation outcome after ACL reconstruction. Med Arch. 2015;69(1):21.

25. Kuenze C, Lisee C, Birchmeier T, et al. Sex differences in quadriceps rate of torque development within 1 year of ACL reconstruction. Phys Ther Sport. 2019;38:36-43.
26. Buchanan PA, Vardaxis VG. Lower-extremity strength profiles and gender-based classification of basketball players ages 9-22 years. J Strength Cond Res. 2009;23(2):406-419.

27. Palmieri-Smith RM, Lepley LK. Quadriceps strength asymmetry after anterior cruciate ligament reconstruction alters knee joint biomechanics and functional performance at time of return to activity. Am J Sport Med. 2015;43(7):1662-1669.

28. Fitzgerald GK, Piva SR, Irrgang JJ. A modified neuromuscular electrical stimulation protocol for quadriceps strength training following anterior cruciate ligament reconstruction. J Orthop Sports Phys Ther. 2003;33(9):492-501.

29. Garrison JC, Hannon J, Goto S, et al. Knee loading after ACL-R is related to quadriceps strength and knee extension differences across the continuum of care. Orthop J Sports Med. 2019;7(10):2325967119870155.

30. Beischer S, Senorski EH, Thomeé C, Samuelsson $\mathrm{K}$, Thomeé R. Knee strength, hop performance and self-efficacy at 4 months are associated with symmetrical knee muscle function in young athletes 1 year after an anterior cruciate ligament reconstruction. Br J Sports Med. 2019;5(1):e000504.

31. Žargi TG, Drobnič M, Vauhnik R, Koder J, Kacin A. Factors predicting quadriceps femoris muscle atrophy during the first 12 weeks following anterior cruciate ligament reconstruction. Knee. 2017;24(2):319-328. 